\title{
IncRNA FAM83H-AS1 is associated with the prognosis of colorectal carcinoma and promotes cell proliferation by targeting the Notch signaling pathway
}

\author{
SONG LU, WEI DONG, PEI ZHAO and ZHENJUN LIU \\ ICU of Sichuan Cancer Hospital and Institute, Sichuan Cancer Center, School of Medicine, \\ University of Electronic Science and Technology of China, Chengdu, Sichuan 610041, P.R. China
}

Received June 27, 2017; Accepted November 23, 2017

DOI: $10.3892 / \mathrm{ol} .2017 .7520$

\begin{abstract}
The long non-coding RNA, FAM83H antisense RNA 1 (head to head) (FAM83H-AS1), has exhibited a functional role as an oncogene in a number of different types of cancer. The aim of the present study was to reveal the dysregulation of FAM83H-AS1 in colorectal carcinoma (CRC) samples and elucidate its underlying associations with the Notch signaling pathway. The expression profiles of FAM83H-AS1 and two Notch signaling-associated molecules, Notch1 and Hes family basic-helix-loop-helix transcription factor 1 (Hes1), were measured by reverse transcription-polymerase chain reaction and western blot analysis. The Pearson $\chi^{2}$ test was employed to evaluate the associations between FAM83H-AS1 expression and clinical features. A statistically significant positive association between the expression levels of FAM83H-AS1 and those of Notch1 or Hes1 in CRC tissues was analyzed by Spearman's correlation analysis. The Kaplan-Meier method was used to compare the overall survival curves between the highly-expressed and low-expressed FAM83H-AS1 groups via a log-rank test. Specific small hairpin RNA was transfected to silence endogenous FAM83H-AS1. MTT and colony formation assays were performed to measure the growth-inhibition effect of silenced FAM83H-AS1. The levels of FAM83H-AS1, Notch1 and Hes1 were significantly increased in CRC samples and cell lines. Cell proliferation was markedly inhibited when FAM83H-AS1 was knocked down and this effect mediated by FAM83H-AS1 could be reversed by Notch1 regulators. Thus, downregulated
\end{abstract}

Correspondence to: Professor Song Lu, ICU of Sichuan Cancer Hospital and Institute, Sichuan Cancer Center, School of Medicine, University of Electronic Science and Technology of China, 55 Fourth Section of Renmin South Road, Chengdu, Sichuan 610041, P.R. China

E-mail: songlu_mee@163.com

Key words: colorectal carcinoma, long non-coding RNA FAM83H antisense RNA 1 (head to head), Notch signal pathway, proliferative
FAM83H-AS1 exhibited an anti-proliferative role in CRC by repressing the Notch signaling pathway.

\section{Introduction}

Colorectal cancer (CRC), as the third commonest malignant tumor globally, has thrown great threaten to public health $(1,2)$. Despite many efforts have been made to improve the efficiency of diagnosis and treatment of CRC, the prognosis still remains unsatisfied. CRC is a multi-step process involving the dyregulation of multiple genes; therefore, investigations on the precise mechanisms underlying the initiation and progression of CRC are essential.

lncRNAs, a class of RNAs longer than 200 nucleotides with limited or no protein-coding ability, have attracting more and more attention of researchers. IncRNAs have been reported to be involved in a variety of biological processes, including tumorigenesis through regulating oncogenes or tumor suppressors at several levels (3-12). For example, Xia et al (13) demonstrated that long noncoding RNA papillary thyroid carcinoma susceptibility candidate 3 (PTCSC3) inhibited proliferation and invasion of glioma cells by suppressing the Wnt/ $\beta$-catenin signaling pathway. Su et al (14) illustrated that long noncoding RNA BLACAT1 indicated a poor prognosis of colorectal cancer and affected cell proliferation by epigenetically silencing p15. Pei et al (15) reported that downregulation of lncRNA CASC2 promoted cell proliferation and metastasis of bladder cancer by activating the Wnt/ $\beta$-catenin signaling pathway. And also many lncRNAs, for instance, ROR, BLACAT1, ANCR, TUG1, are identified to be dysregulated and play critical role in the initial and progression of CRC (14,16-18). FAM83H-AS1 has been identified to play critical roles in lung cancer and breast cancer $(19,20)$. However, its biological function in CRC has not been thoroughly studied. Notch signaling has been reported to play important roles in regulating cell proliferation, differentiation and death (21-23).

In the present study, we revealed that FAM83H-AS1 was upregulated both in CRC tissues and cells for the first time. Further experiments demonstrated that silenced FAM83H-AS1 could suppress cell proliferation and such function was at least partially mediated by regulating Notch signal pathway. 


\section{Materials and methods}

Patients and specimens. Human primary CRC tissues and their paired adjacent tissues were obtained from 40 patients at the Sichuan Cancer Hospital and Institute, Sichuan Cancer Center, School of Medicine, University of Electronic Science and Technology of China. None of these patients had received local or systemic treatment before the operation. All of the tissues were stored at $-80^{\circ} \mathrm{C}$. An experienced pathologist assessed the differentiation grade, pathological stage, grade and nodal status. The clinical pathological parameters involved in the analysis included age, sex, tumor differentiation, distant metastasis, TNM stage and tumor size (defined based on the diameter of tumor). The tumor differentiation is defined as following: Well, tumor cell differentiation close to normal cell; moderately, tumor cell is poorly differentiated but still retain some vestiges of originally tissues; and poor, tumor cell is very poorly differentiated with no vestiges of originally tissues. The TNM classification is defined as fowling: $T$ is defined as primary tumor $\left(\mathrm{T}_{\mathrm{x}}\right.$ : The primary tumor is not determined; $\mathrm{T}_{0}$ : No original tumor evidence; Tis: carcinoma in situ; $\mathrm{T}_{1}$ : Tumor invading tunica submucosa; $\mathrm{T}_{2}$ : Tumor invading intestinal wall muscularis; $\mathrm{T}_{3}$ : Tumor invading serosa or primary tumor located at the colon or rectum without serosa or tumor located at colon or rectum adjacent tissues; $\mathrm{T}_{4}$ : Primary tumor invading through the peritoneal or invading other ogans). $\mathrm{N}$ is defined as regional lymph nodes $\left(\mathrm{N}_{\mathrm{x}}\right.$ : Regional lymph node is not determined; $\mathrm{N}_{0}$ : No regional lymph node metastasis; $\mathrm{N}_{1}: 1-3$ regional lymph nodes metastasis; $\mathrm{N}_{2}: 4$ or more regional lymph nodes metastasis). $\mathrm{M}$ is defined as distant metastasis $\left(\mathrm{M}_{0}\right.$ : No metastasis; $\mathrm{M}_{1}$ : metastasis). TNM stage includes I-IV. All the written informed consents were obtained from patients. The study protocol was approved by the Ethics Committee of the Sichuan Cancer Hospital and Institute, Sichuan Cancer Center, School of Medicine, University of Electronic Science and Technology of China.

Cell culture. All human colonic cancer cell lines including SW480, LoVo, HCT116, HT29 and the human colonic epithelial cells HCoEpiC were obtained from the American Type Culture Collection (ATCC; Manassas, VA, USA). Cells were cultured in RPMI-1640 supplemented with 10\% fetal bovine serum at $37^{\circ} \mathrm{C}$ in a incubator containing $5 \%$ of $\mathrm{CO}_{2}$.

Cell transfection. CRC cells were placed with a desired cell number and $24 \mathrm{~h}$ later, target shRNA and non-targeting controls (shRNA; Dharmacon, Inc., Lafayette, CO, USA) were transfected with a final concentration of $10 \mathrm{nM}$. Lipofectamine RNAiMAX reagent and OptiMEM medium were used for the cell interference assays on the basis of the manufacturer's instructions (Invitrogen, Carlsbad, CA, USA). SMART pool of FAM83H-AS1 shRNA (Dharmacon, Inc.) was used in this study to form silenced FAM83H-AS1 (sh-FAM83H-AS1). The interference efficiency was examined by reverse transcription-quantitative polymerase chain reaction (RT-qPCR). Cell lines stably interfering FAM83H-AS1 were transfected with the plasmid sh-FAM83H-AS1, and screened with G418 $(10 \mathrm{mg} / \mathrm{ml})$ for two months.

$R T$ - $q P C R$. Total RNA was severally extracted from tumor tissues and cell lines by using a Trizol kit (Invitrogen). cDNA was subsequently synthesized from total RNA by using an Omniscript RT kit (Qiagen, Valencia, CA, USA) under the manufacturer's instructions. RT-PCR reaction was conducted on the Mastercycler ep realplex (Eppendorf 2S; Eppendorf, Hamburg, Germany). A 25- $\mu 1$-reaction mixture contained $1 \mu \mathrm{l}$ of cDNA from samples, $12.5 \mu \mathrm{l}$ of $2 \mathrm{X}$ Fast EvaGreen ${ }^{\mathrm{TM}}$ qPCR Master Mix, $1 \mu \mathrm{l}$ primers $(10 \mathrm{mM})$, and $10.5 \mu \mathrm{l}$ of $\mathrm{RNase} / \mathrm{DNa}$ e-free water. The $\mathrm{Ct}$ value was defined as the cycle number at which the fluorescence intensity reached a certain threshold where amplification of each target gene was within the linear region of the reaction amplification curves. Glyceraldheyde 3-phosphate dehydrogenase (GAPDH) was taken as internal controls. Relative mRNA expression of FAM83H-AS1 was calculated by using the $2^{-\Delta \Delta \mathrm{Ct}}$ method. The primers for FAM83H-AS1 were: forward, 5'-TAGGAAACG AGCGAGCCC-3' and reverse, 5'-GCTTTGGGTCTCCCC TTCTT-3'. The primers for GAPDH were: forward, 5'-GGG AGCCAAAAGGGTCAT-3' and reverse, 5'-GAGTCCTTCC ACGATACCAA-3'.

Cell viability. Cell viability was assessed via 3-(4,5-dimethylthiazol-2-yl)-2,5-diphenyl-trtrazolium bromide (MTT) assay. Cells $\left(5 \times 10^{3}\right.$ cells/well) transfected with indicated vector were seeded in a 96-well flat-bottomed plate for $24 \mathrm{~h}$ and cultured in the normal medium. At 0, 24, 48, 72 and $96 \mathrm{~h}$ after transfection, the MTT solution $(5 \mathrm{mg} / \mathrm{ml}, 20 \mu \mathrm{l})$ was added to each well. Following incubation for $4 \mathrm{~h}$, the media was removed and $100 \mu \mathrm{l}$ DMSO was added to each well. The relative number of surviving cells was assessed by measuring the optical density (OD) of cell lysates at $560 \mathrm{~nm}$. All assays were performed in triplicate.

5-ethynyl-2'-deoxyuridine (EdU) proliferation assay. The EdU proliferation assay was conducted using Cell-Light EdU Apollo 567 In Vitro Imaging kit (RiboBio, Guangzhou, China) under the introduction of the manufracture's instructions. Briefly, approximately $5 \times 10^{3}$ cells/wells with indicated treatments were seeded into 96-well plates. After $24 \mathrm{~h}, 100 \mu \mathrm{l}$ medium with $50 \mu \mathrm{M}$ EdU was added into each well and incubated for $2 \mathrm{~h}$ under $37^{\circ} \mathrm{C}$. Afterwards, cells were fixed with 4\% paraformaldehyde, then stained with Hoechst 33342 and Apollo reaction cocktail. Images were taken by applying fluorescence microscopy (Nikon Corporation, Tokyo, Japan) and then merged by Photoshop 6.0 software. EdU-positive cells and total cells were counted within each field.

Colony formation assay. Cells (500 cells/well) transfected with indicated vector were plated in 6-well plates and incubated at $37^{\circ} \mathrm{C}$. Two weeks later, the cells were fixed and stained with $0.1 \%$ of crystal violet. The number of visible colonies was counted manually.

Western bolt analysis and antibodies. Total protein lysates were separated in $10 \%$ of sodium dodecyl sulfate-polyacrylamide gel electrophoresis (SDS-PAGE), and were electrophoretically transferred to polyvinylidene difluoride membranes (Roche Diagnostics GmbH, Mannheim, Germany). Protein loading was estimated by using mouse anti-GAPDH monoclonal antibody. The membranes were blotted with $10 \%$ of non-fat milk in TBST for $2 \mathrm{~h}$ at room temperature, washed and then probed with 

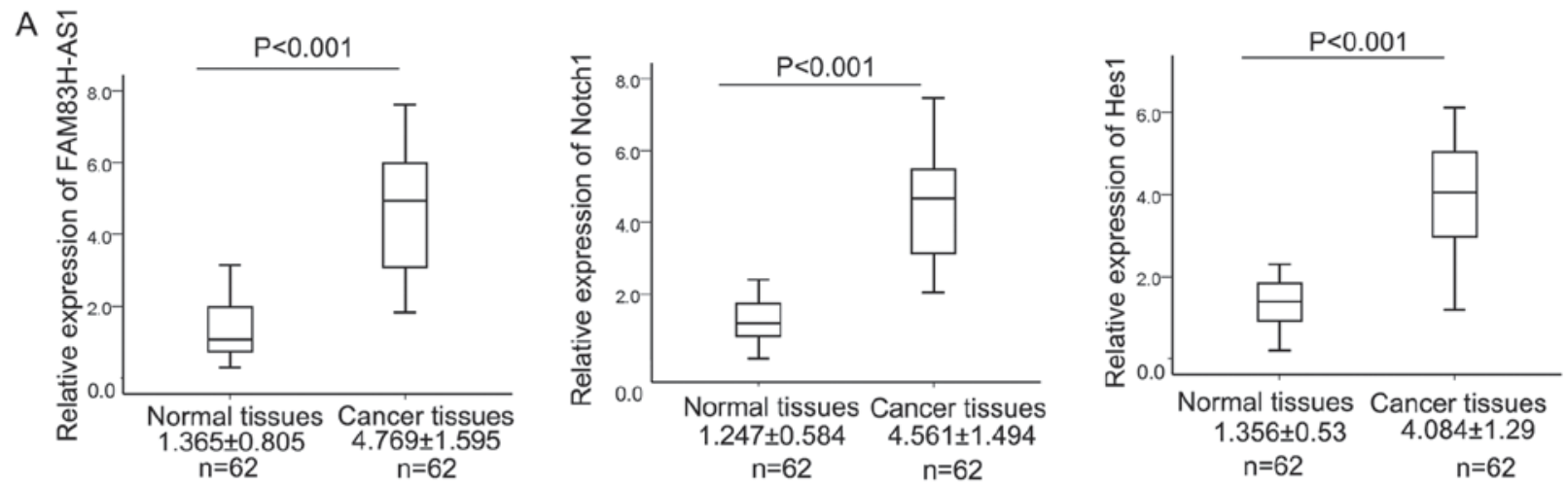

B
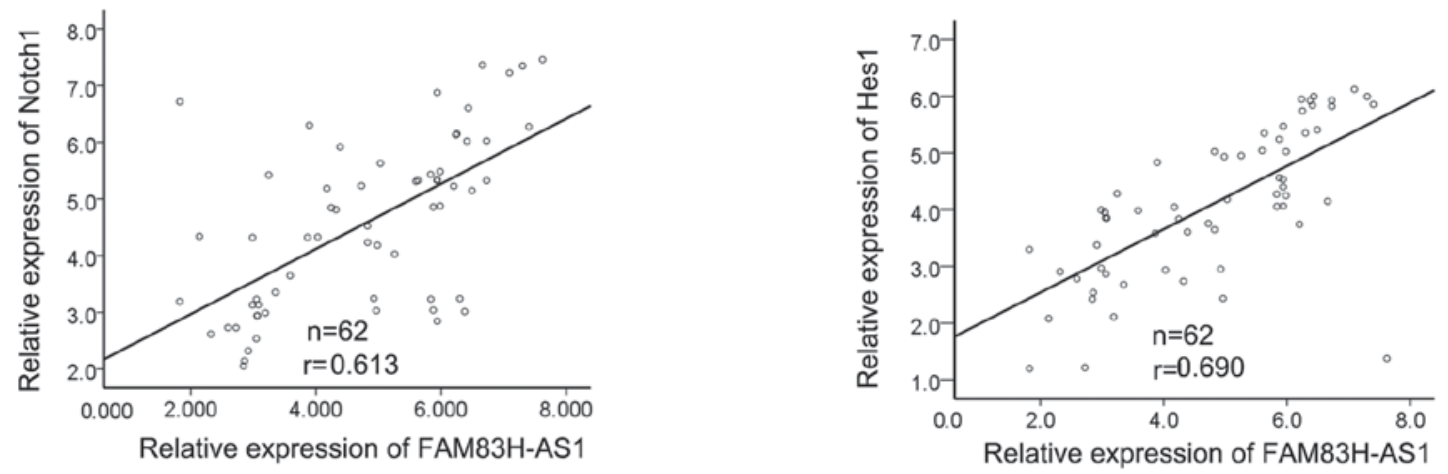

$\mathrm{C}$
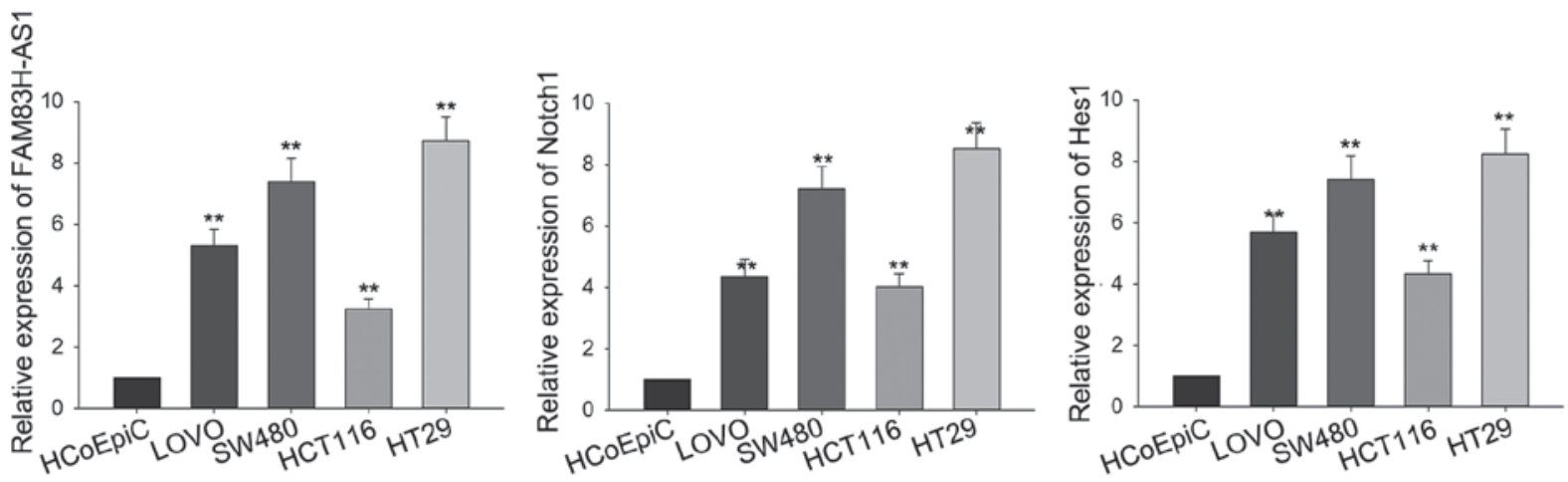

Figure 1. FAM83H-AS1 and Notch1 were upregulated in CRC tissues and cell lines. (A) The levels of FAM83H-AS1, Notch1 and Hes1 in CRC tissues and the corresponding normal tissues were measured by RT-qPCR. (B) The correlation of FAM83H-AS1 with Notch1 and Hes1 was analyzed by Spearman's correlation analysis. (C) The levels of FAM83H-AS1, Notch1 and Hes1 were also determined by RT-qPCR in four human colonic cancer cell lines including SW480, LoVo, HCT116, HT29, and the human colonic epithelial cell HCoEpiC (control). Data are presented as the mean \pm standard deviation of at least three independent experiments. ${ }^{* *}$ P $<0.01$ vs. control group. FAM83H-AS1, FAM83H antisense RNA 1 (head to head); CRC, colorectal carcinoma; RT-qPCR, reverse transcription-quantitative polymerase chain reaction; Hes1, Hes family basic-helix-loop-helix transcription factor 1.

the rabbit anti-Notch1 (1:2,000 dilution), Hes1 (1:2,000 dilution), and GAPDH (1:3,000 dilution), overnight $(8 \mathrm{~h})$ at $4^{\circ} \mathrm{C}$, followed by treatment with secondary antibody conjugated to horseradish peroxidase for $2 \mathrm{~h}$ at room temperature. The proteins were detected by using an enhanced chemiluminescence system and exposed to X-ray film. All antibodies were purchased from Abcam (Cambridge, MA, USA).

Statistical analysis. Data were shown as the means \pm standard error of at least three independent experiments. The SPSS 17.0 software (SPSS, Inc., Chicago, IL, USA) was used for statistical analysis. Two group comparisons were performed with a Student's t-test. Multiple group comparisons were analyzed with one-way ANOVA. Statistically significant positive correlation between the expression level of FAM83H-AS1 and that of Notch1 or Hes1 in CRC tissues was analyzed by Spearman's correlation analysis. The Pearson $\chi^{2}$ test was used to evaluate the relationship between the expression of FAM83H-AS1 and clinical features. Kaplan-Meier method was used to compare the overall survival curves between high-FAM83H-AS1 and low-FAM83H-AS1 expression groups via the log-rank test. $\mathrm{P}<0.05$ was considered to indicate a statistically significant difference.

\section{Results}

FAM83H-AS1 and Notch1 were upregulated in CRC tissues and cell lines. To explore the biological function of FAM83H-AS1 and its relationship with Notch signaling in $\mathrm{CRC}$, we first measured the levels of FAM83H-AS1, Notch1 and Hes1 in the CRC tissues and corresponding normal tissues by RT-qPCR. As shown in Fig. 1A, compared with corresponding normal 
Table I. Associations between FAM83H antisense RNA 1 (Head to Head) expression and clinical features.

\begin{tabular}{|c|c|c|c|}
\hline \multirow[b]{2}{*}{ Variable } & \multicolumn{2}{|c|}{$\begin{array}{l}\text { FAM83H-AS1 } \\
\text { expression }\end{array}$} & \multirow[b]{2}{*}{ P-value } \\
\hline & Low (n) & $\operatorname{High}(\mathrm{n})$ & \\
\hline Sex & & & 0.310 \\
\hline Male & 18 & 13 & \\
\hline Female & 13 & 18 & \\
\hline Age (years) & & & 0.124 \\
\hline$<60$ & 10 & 17 & \\
\hline$\geq 60$ & 21 & 14 & \\
\hline Tumor differentiation & & & 0.309 \\
\hline Well/moderately & 12 & 17 & \\
\hline Poorly & 19 & 14 & \\
\hline Distant metastasis & & & 0.202 \\
\hline No & 14 & 20 & \\
\hline Yes & 17 & 11 & \\
\hline TNM stages & & & $0.004^{\mathrm{a}}$ \\
\hline I-II & 24 & 12 & \\
\hline III-IV & 7 & 19 & \\
\hline Tumor size & & & $0.002^{\mathrm{a}}$ \\
\hline$<5 \mathrm{~cm}$ & 24 & 11 & \\
\hline$\geq 5 \mathrm{~cm}$ & 7 & 20 & \\
\hline
\end{tabular}

Low and high expression groups were determined by the sample median. Mean age was $60.88 \pm 9.5$ ( $\mathrm{n}=62$ patients). ${ }^{\mathrm{a}} \mathrm{P}<0.01$. FAM83H-AS1, FAM83H antisense RNA 1 (head to head). TNM, tumor-node-metastasis staging.

tissues, the levels of FAM83H-AS1, Notch1 and Hes1 were significantly increased in CRC tissues. And the FAM83H-AS1 showed significantly positive correlation with Notch1 and Hes1, analyzed by Spearman's correlation analysis (Fig. 1B). Then, the levels of FAM83H-AS1, Notch1 and Hes1 were also determined in four human colonic cancer cell lines including SW480, LoVo, HCT116, HT29 and a human colonic epithelial cell HCoEpiC. As presented in Fig. 1C, the expression levels of the three molecules were obviously increased in all the four $\mathrm{CRC}$ cell lines in comparison to the human colonic epithelial cell. The consistent expression levels of FAM83H-AS1, Notch1 and Hes1 both in CRC tissues and cell lines indicated that FAM83H-AS1 might be involved in the progression of CRC and implied the association between FAM83H-AS1 and Notch signal pathway.

High level of FAM83H-AS1 was associated with clinical features and poor prognosis. To detect the clinical significance of FAM83H-AS1 expression in CRC, 62 patients were divided into highly-expressed FAM83H-AS1 group $(n=31)$ and low-expressed FAM83H-AS1 group $(n=31)$ according to the cut-off value, which was defined as the median of the cohort. As revealed in Table I, high expression of FAM83H-AS1 in $\mathrm{CRC}$ patients was significantly correlated with advanced tumor stage $(\mathrm{P}=0.004)$ and large tumor size $(\mathrm{P}=0.002)$. In addition,

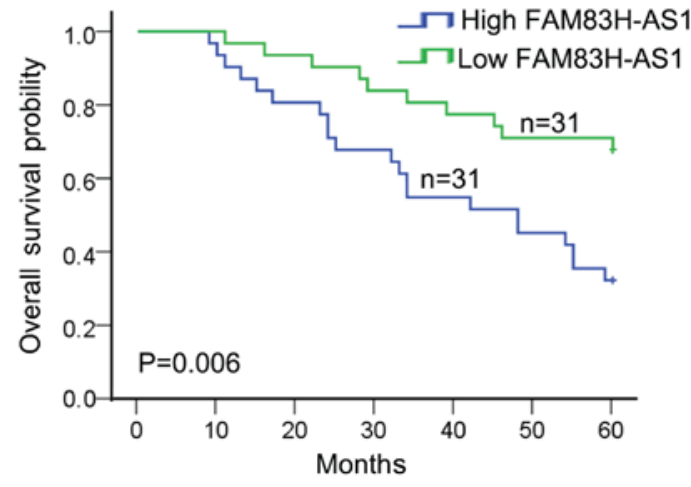

Figure 2. Associations between FAM83H-AS1 and prognosis of colorectal carcinoma was analyzed using the Kaplan-Meier method (log-rank test). FAM83H-AS1, FAM83H antisense RNA 1 (head to head).

patients with high expression of FAM83H-AS1 were associated with worse overall survival in CRC patients ( $\mathrm{P}=0.006$; Fig. 2). These data indicated that FAM83H-AS1 might act as a potent biomarker for predicting prognosis in CRC patients.

Silenced FAM83H-AS1 inhibited cell proliferation and migration via affecting cell apoptosis. To investigate the function of FAM83H-AS1 on the cell proliferation of CRC cells, SW480 and HT29 cells were transfected with sh-FAM83H-AS1. Satisfied transfection efficiency was obtained after $48 \mathrm{~h}$ (Fig. 3A). MTT assay was performed to measure the viability of SW480 and HT29 cells transfected with sh-FAM83H-AS1, and results illustrated that silenced FAM83H-AS1 could significantly inhibit the cell proliferation ability (Fig. 3B). The result of colony formation assays indicated that colony formation was remarkably inhibited by FAM83H-AS1 knockdown (Fig. 3C). Besides, the inhibited cell proliferation by sh-FAM83H-AS1 was further affirmed by the data from EdU assay (Fig. 3D). Furthermore, transwell assay was designed to detect how silenced FAM83H-AS1 affected cell migration. As diagramed in Fig. 3E, the knockdown of FAM83H-AS1 remarkably impaired migratory cells in comparison with the negative control. Meanwhile, the silence of FAM83H-AS1 also augmented the apoptotic cells compared to the negative control (Fig. 3F). These data indicated that knockdown FAM83H-AS1 suppressed the proliferative and migratory ability of CRC cells through influencing cell apoptosis.

The growth-inhibition in CRC cells mediated by the knockdown of FAM83H-AS1 could be reversed by Notch1 activator. It has been implicated that Notch signal pathway was involved in regulating cell proliferation $(24,25)$. Due to the correlation of FAM83H-AS1 with Notch1 and its downstream gene Hes1, we hypothesized that the function of FAM83H-AS1 in CRC might be mediated by Notch signal pathway. To confirm this hypothesis, we measured the level of Notch in response to the knockdown of FAM83H-AS1. As illustrated in Fig. 4A, silenced FAM83H-AS1 could suppress the expressions of Notch1 and Hest1 both in mRNA and protein levels. To make further confirmation, Jagged-1/Fc, one Notch1 activator, was added to activate the Notch signal pathway. After Jagged-1/Fc was added, the weakened expressions of Notch1 ans Hes1 triggered by the silence of FAM83H-AS1 were recovered a 
A
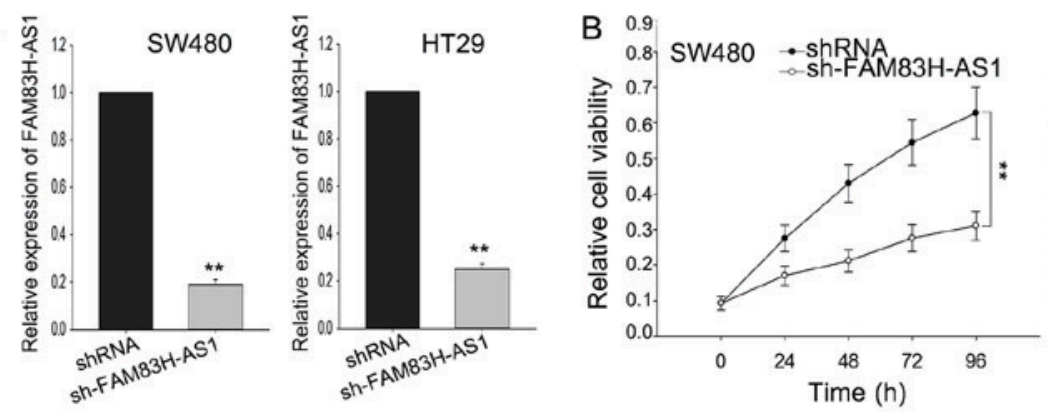

C
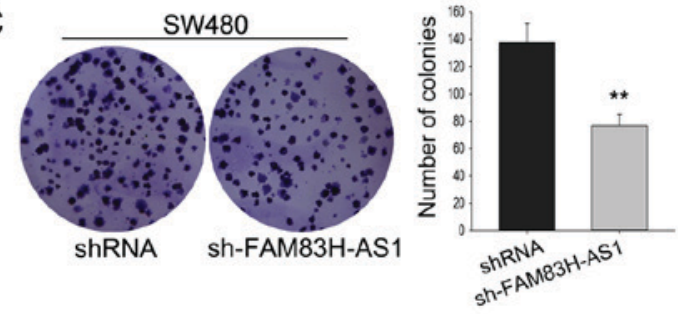

D

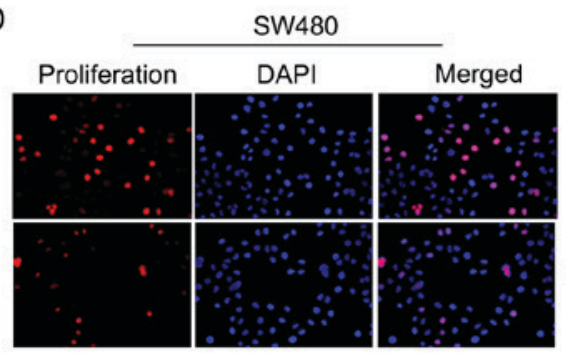

E

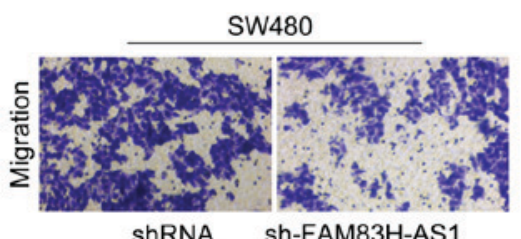

shRNA sh-FAM83H-AS1

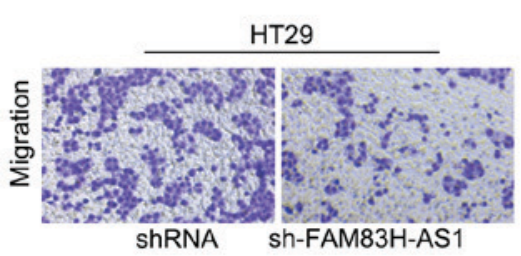

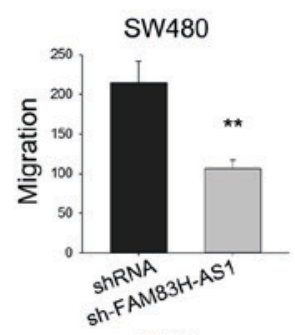

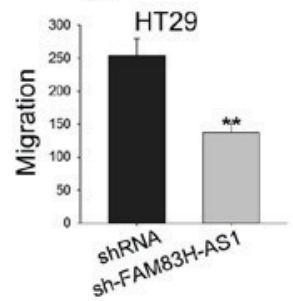

sh-FAM83H-AS1

F
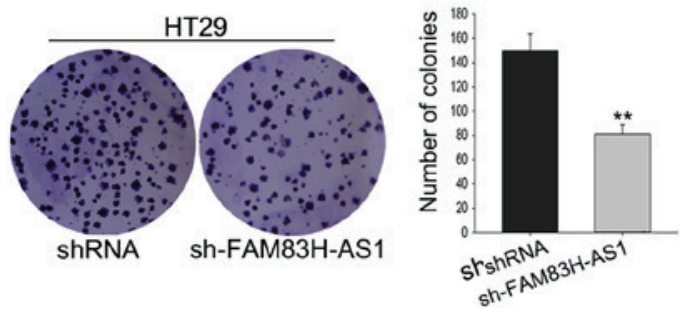
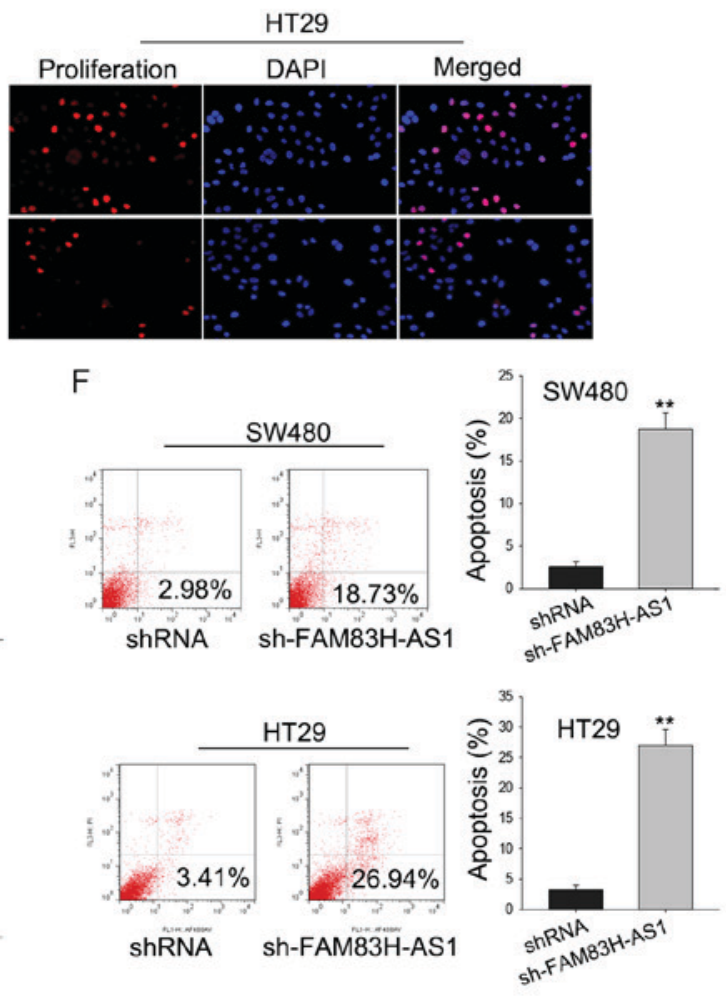

Figure 3. Silenced FAM83H-AS1 inhibited cell proliferation and migration via cell apoptosis. (A) SW480 and HT29 cells were transfected with sh-FAM83H-AS1, and satisfied transfection efficiency was obtained by reverse transcription-quantitative polymerase chain reaction. The effect of silenced FAM83H-AS1 on cell viability and proliferation ability was measured by (B) MTT and (C) colony formation assays as well as (D) EdU assay. (E) A Transwell assay was used to measure the effect of silenced FAM83H-AS1 on cell migration. (F) Flow cytometry analysis was applied to examine how the FAM83H-AS1 knockdown influenced cell apoptosis. Data are presented as the mean \pm standard deviation of at least three independent experiments. Scale bars, $200 \mu \mathrm{m}$. ${ }^{* *} \mathrm{P}<0.01 \mathrm{vs.} \mathrm{control}$ group. FAM83H-AS1, FAM83H antisense RNA 1 (head to head); sh, small hairpin.

little (Fig. 4B). Additionally, as presented in Fig. 4C and D, results from $\mathrm{MTT}$ and colony formation revealed that the growth-inhibition effect mediated by silenced FAM83H-AS1 could be reversed by the addition of Jagged-1/Fc. These findings revealed that the function of FAM83H-AS1 exerted in $\mathrm{CRC}$ was at least partially mediated by Notch signal pathway.

The effect of FAM83H-AS1 on CRC cells was dependent on Notch pathway. To determine whether Notch signal pathway was essential for FAM83H-AS, we applied N-[N-(3,5difluorophenacetyl)-L-alanyl]-S-phenylglycine t-butyl ester (DAPT), a widely used $\gamma$-secretase inhibitor (GSI) which could effectively repress the expression of receptors and ligands in Notch signaling pathway, to inhibit Notch-1 expression in our study and then we measured the effect of FAM83H-AS1 knockdown on cell proliferation. As presented in Fig. 5A, after cells were treated with DAPT, the level of Notch-1 was significantly reduced. Subsequently, we observed how the expression 

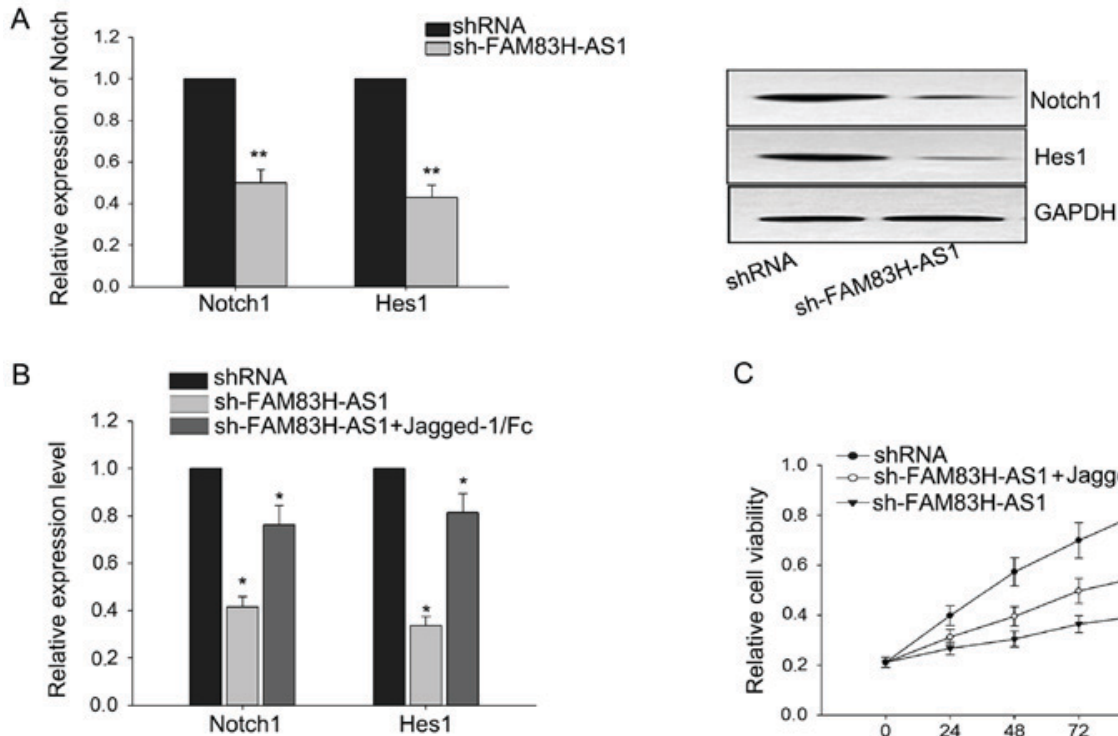

C
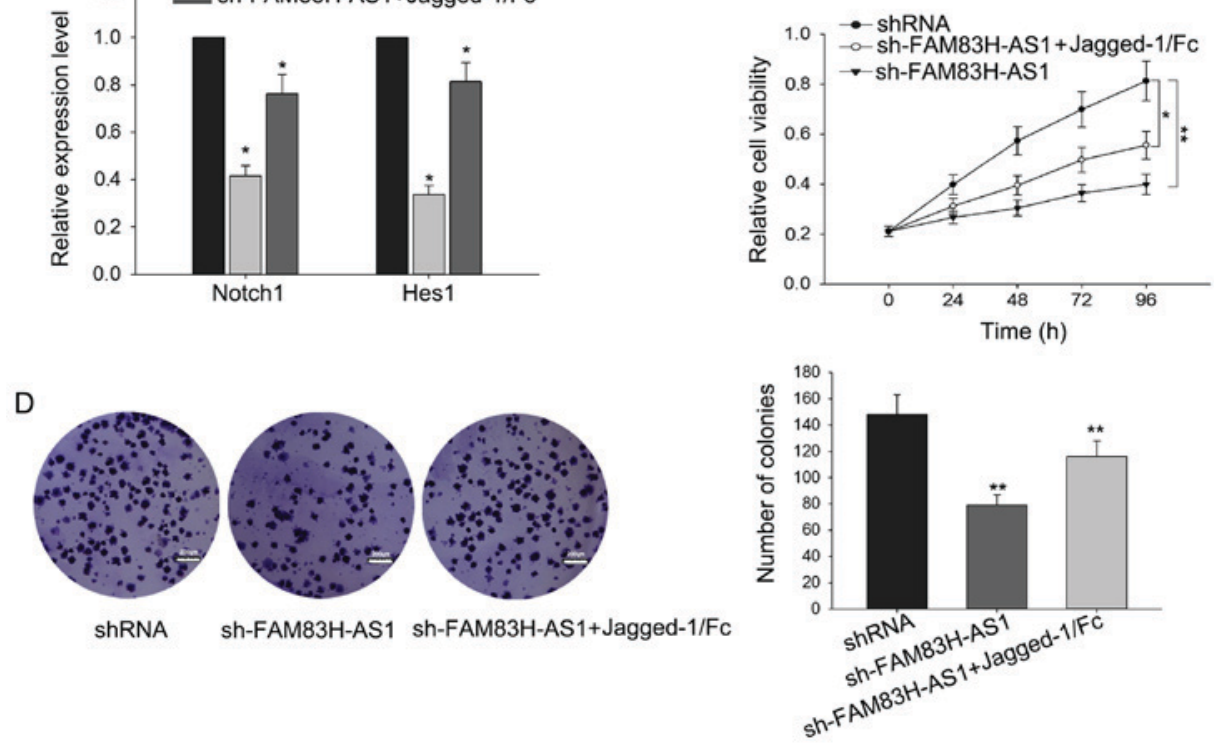

Figure 4. Growth inhibition in CRC cells mediated by the knockdown of FAM83H-AS1 was reversed by the Notch1 activator. (A) The expressions of Notch1 and Hes1 at the mRNA and protein levels were detected by western blotting and reverse transcription-quantitative polymerase chain reaction when cells were transfected with sh-FAM83H-AS1. (B) Rescue assays were employed to detect the expressions of Notch1 and Hes1 following the silence of FAM83H-AS1 and the addition of Jagged-1/Fc. (C) MTT and (D) colony formation assays were performed to measure the viability and growth ability of cells treated with sh-FAM83H-AS1 and Jagged-1/Fc. Data are presented as the mean \pm standard deviation of at least three independent experiments. Scale bars, $200 \mu \mathrm{m}$. ${ }^{*} \mathrm{P}<0.05$ and ${ }^{* *} \mathrm{P}<0.01$ vs. control group. CRC, colorectal carcinoma; FAM83H-AS1, FAM83H antisense RNA 1 (head to head); sh, small hairpin; Hes1, Hes family basic-helix-loop-helix transcription factor 1.
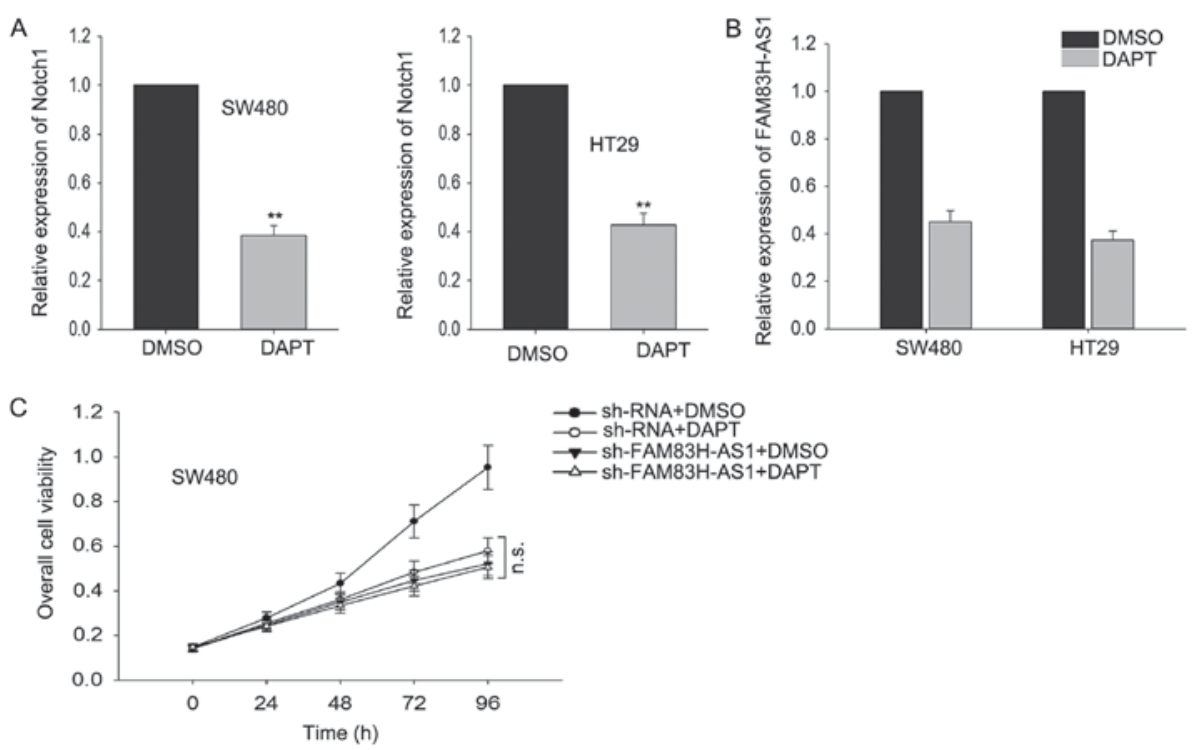

Figure 5. Effect of FAM83H-AS1 on CRC cells was dependent on the Notch signaling pathway. (A) The level of Notch1 was silenced by DAPT in CRC cells based on reverse transcription-quantitative polymerase chain reaction. (B) The effect of silenced Notch1 on the expression level of FAM83H-AS1. (C) Rescue assays were applied to determine the unchanged proliferation of CRC cells following the silencing of Notch1 and FAM83H-AS1 induced by DAPT. Data are presented as the mean \pm standard deviation of at least three independent experiments. ${ }^{* *} \mathrm{P}<0.01$ vs. DMSO (control) group. DAPT, $\mathrm{N}$-[N-(3,5-difluorophenacetyl)-L-alanyl]-S-phenylglycine t-butyl ester; CRC, colorectal carcinoma; FAM83H-AS1, FAM83H antisense RNA 1 (head to head); sh, small hairpin. 
level of FAM83H-AS1 responded to the silenced Notch1 by DAPT, and discovered that silenced Notch1 resulted in the knockdown of FAM83H-AS1 (Fig. 5B). Then we performed MTT assays and found that after Notch-1 was suppressed, the effect induced by the knockdown of FAM83H-AS1 was vanished (Fig. 5C). Our results indicated that FAM83H-AS1 worked through the Notch pathway.

\section{Discussion}

lncRNAs, a class of RNAs longer than 200 nucleotides with limited or no protein-coding ability, have attracting more and more attention of researchers. Currently, accumulating lncRNAs have been identified in different biological processes, in which they act as critical regulators in both physiology and pathology, including tumorigenesis. Idogawa et al (26) reported that IncRNA NEAT1 was a transcriptional target of p53 and modulated p53-induced transactivation and tumor-suppressor function. Zhao et al (27) revealed that SNHG5/miR-32 axis regulated cell proliferation and migration of gastric cancer by targeting KLF4. Malakar et al (28) demonstrated that long noncoding RNA MALAT1 promoted hepatocellular carcinoma development by upregulating SRSF1 and activating mTOR. As for CRC, plenty well-studied lncRNAs have been reported to be dysregulated in CRC tissues and participate in CRC progression via multiple mechanisms (29-31). FAM83H-AS1 has been identified to play critical roles in lung cancer and breast cancer $(19,20)$. However, its biological function in CRC has not been studied.

In our present study, we demonstrated for the first time that lncRNA FAM83H-AS1 was upregulated in CRC tissues and cell lines. Further functional studies revealed that silenced FAM83H-AS1 could inhibit cell proliferation and migration through inducing more apoptosis. Furthermore, concomitant upregulation of two Notch signaling molecules, Notch1 and Hes1, was obtained in both CRC tissues and cell lines, indicating the potential mechanism of FAM83H-AS1/Notch signal pathway in CRC. Notch signaling pathway has been reported to involve in the tumorigenesis of various cancer types $(23,32,33)$. In our study, we showed the accompanied activation of Notch signaling in CRC tissues and cells and rescue assays revealed that the growth inhibition mediated by the knockdown of FAM83H-AS1 was at least in a Notch signal dependent manner, which was further confirmed by the effect of DAPT on Notch1 and FAM83H-AS1 as well as the unchanged cell proliferation after Notch1 was silenced by DAPT. All in all, despite the fact that we have made progress in studying the initiation and development of CRC to some extent, our research just takes a little part in the mechanisms of the tumorigenesis of CRC. Therefore, this paper has not been involved in the study about the fact that cadmium prolonged exposure involved malignant progression of A549 cells. And as for the situation above, we will put much more attention in the future study upon the research that whether the hot spicy eating habit of people in Sichuan is related to the tumorigenesis of CRC.

In conclusion, FAM83H-AS1 was demonstrated to be upregulated in both CRC tissues and cell lines, wherein acting as an oncogene via activating Notch signaling. Due to the complicated cross talk of Notch signaling with various tumorigenesis regulators, still many studies need to be made to further investigate the Notch1-mediated mechanism under
FAM83H-AS1 dysfunction, for exploring the potential therapeutic value of FAM83H-AS1 in treating CRC.

\section{References}

1. Booth RA: Minimally invasive biomarkers for detection and staging of colorectal cancer. Cancer Lett 249: 87-96, 2007.

2. Jemal A, Bray F, Center MM, Ferlay J, Ward E and Forman D: Global cancer statistics. CA Cancer J Clin 61: 69-90, 2011.

3. Zhu M, Liu J, Xiao J, Yang L, Cai M, Shen H, Chen X, Ma Y, $\mathrm{Hu} \mathrm{S}$, Wang Z, et al: Lnc-mg is a long non-coding RNA that promotes myogenesis. Nat Commun 8: 14718, 2017.

4. Zhou X, Yuan P, Liu Q and Liu Z: LncRNA MEG3 regulates imatinib resistance in chronic myeloid leukemia via suppressing microRNA-21. Biomol Ther (Seoul) 25: 490-496, 2017.

5. Zhou C, Huang C, Wang J, Huang H, Li J, Xie Q, Liu Y, Zhu J, Li Y, Zhang D, et al: LncRNA MEG3 downregulation mediated by DNMT3b contributes to nickel malignant transformation of human bronchial epithelial cells via modulating PHLPP1 transcription and HIF-1 $\alpha$ translation. Oncogene 36: 3878-3889, 2017.

6. Zhang H, Xiong Y, Xia R, Wei C, Shi X and Nie F: The pseudogene-derived long noncoding RNA SFTA1P is down-regulated and suppresses cell migration and invasion in lung adenocarcinoma. Tumour Biol 39: 1010428317691418, 2017.

7. Zhang D, Sun G, Zhang H, Tian J and Li Y: Long non-coding RNA ANRIL indicates a poor prognosis of cervical cancer and promotes carcinogenesis via PI3K/Akt pathways. Biomed Pharmacother 85: 511-516, 2017.

8. Zhang CZ: Long non-coding RNA FTH1P3 facilitates oral squamous cell carcinoma progression by acting as a molecular sponge of miR-224-5p to modulate fizzled 5 expression. Gene 607: 47-55, 2017.

9. Zhang CL, Zhu KP and Ma XL: Antisense lncRNA FOXC2-AS1 promotes doxorubicin resistance in osteosarcoma by increasing the expression of FOXC2. Cancer Lett 396: 66-75, 2017.

10. Yu H, Xue Y, Wang P, Liu X, Ma J, Zheng J, Li Z, Li Z, Cai H and Liu Y: Knockdown of long non-coding RNA XIST increases blood-tumor barrier permeability and inhibits glioma angiogenesis by targeting miR-137. Oncogenesis 6: e303, 2017.

11. Yang C, Li Z, Li Y, Xu R, Wang Y, Tian Y and Chen W: Long non-coding RNA NEAT1 overexpression is associated with poor prognosis in cancer patients: A systematic review and meta-analysis. Oncotarget 8: 2672-2680, 2017.

12. $\mathrm{Xu} \mathrm{J}$, Zhang $\mathrm{R}$ and Zhao J: The novel long noncoding RNA TUSC7 inhibits proliferation by sponging miR-211 in colorectal cancer. Cell Physiol Biochem 41: 635-644, 2017.

13. Xia S, Ji R and Zhan W: Long noncoding RNA papillary thyroid carcinoma susceptibility candidate 3 (PTCSC3) inhibits proliferation and invasion of glioma cells by suppressing the Wnt/ $\beta$-catenin signaling pathway. BMC Neurol 17: 30, 2017.

14. Su J, Zhang E, Han L, Yin D, Liu Z, He X, Zhang Y, Lin F, Lin Q, Mao P, et al: Long noncoding RNA BLACAT1 indicates a poor prognosis of colorectal cancer and affects cell proliferation by epigenetically silencing of p15. Cell Death Dis 8: e2665, 2017.

15. Pei Z, Du X, Song Y, Fan L, Li F, Gao Y, Wu R, Chen Y, Li W, Zhou H, et al: Down-regulation of IncRNA CASC2 promotes cell proliferation and metastasis of bladder cancer by activation of the Wnt/ $\beta$-catenin signaling pathway. Oncotarget 8: 18145-18153, 2017.

16. Li H, Jiang X and Niu X: Long non-coding RNA reprogramming (ROR) promotes cell proliferation in colorectal cancer via affecting p53. Med Sci Monit 23: 919-928, 2017.

17. Yang ZY, Yang F, Zhang YL, Liu B, Wang M, Hong X, Yu Y, Zhou $\mathrm{YH}$ and Zeng $\mathrm{H}$ : LncRNA-ANCR down-regulation suppresses invasion and migration of colorectal cancer cells by regulating EZH2 expression. Cancer Biomark 18: 95-104, 2017.

18. Wang L, Zhao Z, Feng W, Ye Z, Dai W, Zhang C, Peng J and Wu K: Long non-coding RNA TUG1 promotes colorectal cancer metastasis via EMT pathway. Oncotarget 7: 51713-51719, 2016.

19. Zhang J, Feng S, Su W, Bai S, Xiao L, Wang L, Thomas DG, Lin J, Reddy RM, Carrott PW, et al: Overexpression of FAM83H-AS1 indicates poor patient survival and knockdown impairs cell proliferation and invasion via MET/EGFR signaling in lung cancer. Sci Rep 7: 42819, 2017.

20. Yang F, Lv SX, Lv L, Liu YH, Dong SY, Yao ZH, Dai XX, Zhang XH and Wang OC: Identification of IncRNA FAM83H-AS1 as a novel prognostic marker in luminal subtype breast cancer. Onco Targets Ther 9: 7039-7045, 2016. 
21. Xiao W, Gao Z, Duan Y, Yuan W and Ke Y: Notch signaling plays a crucial role in cancer stem-like cells maintaining stemness and mediating chemotaxis in renal cell carcinoma. J Exp Clin Cancer Res 36: 41, 2017.

22. Teoh SL and Das S: Notch signalling pathways and their importance in the treatment of cancers. Curr Drug Targets: Mar 9, 2017 (Epub ahead of print).

23. Gao J, Long B and Wang Z: Role of Notch signaling pathway in pancreatic cancer. Am J Cancer Res 7: 173-186, 2017.

24. Gotte M, Greve B, Kelsch R, Müller-Uthoff H, Weiss K, Kharabi Masouleh B, Sibrowski W, Kiesel L and Buchweitz O: The adult stem cell marker Musashi-1 modulates endometrial carcinoma cell cycle progression and apoptosis via Notch-1 and p21WAF1/CIP1. Int J Cancer 129: 2042-2049, 2011

25. Guo Q, Qian Z, Yan D, Li L and Huang L: LncRNA-MEG3 inhibits cell proliferation of endometrial carcinoma by repressing Notch signaling. Biomed Pharmacother 82: 589-594, 2016.

26. Idogawa M, Ohashi T, Sasaki Y, Nakase $H$ and Tokino $T$ : Long non-coding RNA NEAT1 is a transcriptional target of p53 and modulates p53-induced transactivation and tumor-suppressor function. Int J Cancer 140: 2785-2791, 2017.

27. Zhao L, Han T, Li Y, Sun J, Zhang S, Liu Y, Shan B, Zheng D and Shi J: The lncRNA SNHG5/miR-32 axis regulates gastric cancer cell proliferation and migration by targeting KLF4. FASEB J 31 : 893-903, 2017.
28. Malakar P, Shilo A, Mogilavsky A, Stein I, Pikarsky E, Nevo Y, Benyamini H, Elgavish S, Zong X, Prasanth KV and Karni R: Long noncoding RNA MALAT1 promotes hepatocellular carcinoma development by SRSF1 upregulation and mTOR activation. Cancer Res 77: 1155-1167, 2017.

29. Peng W, Wang $Z$ and Fan H: LncRNA NEAT1 impacts cell proliferation and apoptosis of colorectal cancer via regulation of Akt signaling. Pathol Oncol Res 23: 651-656, 2017.

30. Ye Z, Zhou M, Tian B, Wu B and Li J: Expression of IncRNA-CCAT1, E-cadherin and N-cadherin in colorectal cancer and its clinical significance. Int J Clin Exp Med 8: 3707-3715, 2015.

31. Xiang JF, Yin QF, Chen T, Zhang Y, Zhang XO, Wu Z, Zhang S, Wang HB, Ge J, Lu X, et al: Human colorectal cancer-specific CCAT1-L lncRNA regulates long-range chromatin interactions at the MYC locus. Cell Res 24: 513-531, 2014.

32. Fujiki K, Inamura H, Miyayama T and Matsuoka M: Involvement of Notch1 signaling in malignant progression of A549 cells subjected to prolonged cadmium exposure. J Biol Chem 292: 7942-7953, 2017.

33. Badenes M, Trindade A, Pissarra H, Lopes-da-Costa L and Duarte A: Erratum to: Delta-like 4/Notch signaling promotes $A p c^{\mathrm{Min} / \mathrm{t}}$ tumor initiation through angiogenic and non-angiogenic related mechanisms. BMC Cancer 17: 205, 2017. 